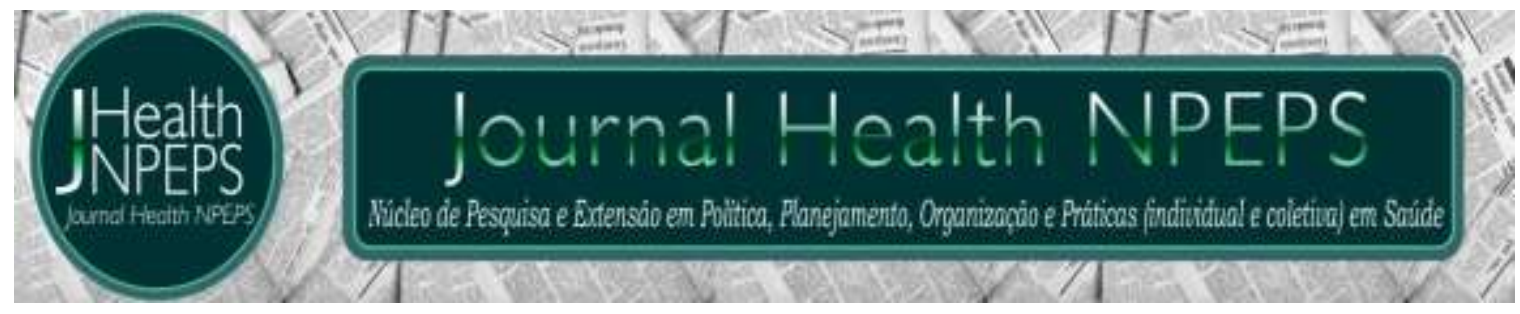

ARTIGO ORIGINAL

\title{
ESTRÉS ACADÉMICO Y CONSUMO DE ALCOHOL EN UNIVERSITARIOS DE NUEVO INGRESO
}

\section{ACADEMIC STRESS AND ALCOHOL CONSUMPTION IN NEW INCOME UNIVERSITIES}

\section{ESTRESSE ACADÊMICO E CONSUMO DE ÁLCOOL EM UNIVERSITÁRIOS DE NOVA ENTRADA}

\begin{abstract}
Laura Hinojosa García1 ${ }^{1}$, Edilaine Cristina da Silva Gherardi-Donato², María Magdalena Alonso Castillo ${ }^{3}$, Raquel Cocenas da Silva ${ }^{4}$, Pedro García García ${ }^{5}$, Viviana Maldonado García 6
\end{abstract}

\section{RESUMEN}

Objetivo: determinar los niveles de estrés académico y el consumo de alcohol en estudiantes universitarios de nuevo ingreso. Método: estudio descriptivo y transversal. Muestreo no probabilístico por conveniencia, para una muestra de 205 alumnos de ambos sexos. Los datos fueron colectados durante el periodo del primero al 15 de diciembre del 2015 y su análisis se realizó por medio del paquete estadístico SPSS versión 22. Resultados: la media de edad de los participantes fue de 19.1 años $(D E=1.86)$, predominó el sexo femenino con un $82.0 \%$ (168). Un $25.4 \%$ (52) menciono estudiar y trabajar. El 65.3\% del total de la muestra reportó niveles de estrés de moderado a alto. La prevalencia del consumo de alcohol en el último año y último mes fue de 59.0 y $32.2 \%$ respectivamente. Un $27.5 \%$ del total de la muestra reportó consumo dependiente, seguido del consumo dañino $16.6 \%$. Conclusión: se identificaron niveles importantes de estrés en los universitarios, además de alta prevalencia de consumo de alcohol. Aunado a esto, un importante porcentaje de los encuestados consume alcohol de forma dependiente, lo cual requiere implementar intervenciones en esta población dirigidas a disminuir el estrés académico, así como abandonar y/o disminuir el consumo de alcohol.

Descriptores: Estudiantes Universitarios; Estrés Académico; Consumo de Alcohol.

\section{ABSTRACT}

Objective: to determine academic stress levels and alcohol consumption in first year university students. Method: descriptive, cross-sectional study. nonprobabilistic convenience sample of 205 students of both sexes. The data were

\footnotetext{
${ }^{1}$ Maestra en Ciencias de Enfermería. Profesor de tiempo completo UAT. E-mail: laura2hg@hotmail.com. Autor principal - Dirección de envío: Neptuno 5-23 col Alianza CP 87000 Matamoros Tamps, México.

${ }^{2}$ PhD Enfermería. Profesor asociado del Departamento de Enfermería Psiquiátrica y Ciencias humanas, Escuela de Enfermería, Universidad de Sao Paulo, Ribeirão Preto, Brasil. E-mail: nane@eerp.usp.br

${ }^{3}$ Dra. en Filosofía. Subdirectora de Posgrado e Investigación, Facultad de Enfermería Universidad Autónoma de Nuevo León, Monterrey Nuevo León, México. E-mail: magdalena_alonso@hotmail.com

${ }^{4} \mathrm{PhD}$ en Ciencias, Departamento de Enfermería Psiquiátrica y Ciencias humanas, Escuela de Enfermería, Universidad de Sao Paulo, Ribeirão Preto, Brasil. E-mail: raquelcocenas@hotmail.com

${ }^{5}$ Maestro en Ciencias de Enfermería, Profesor de Tiempo Completo, Universidad Autónoma de Tamaulipas Unidad Académica Multidisciplinaria Matamoros Tamaulipas, México. E-mail: pedro-2122@hotmail.com

${ }^{6}$ Estadística. Comisión Nacional para el Desarrollo y vida sin Drogas - DEVIDA. E-mail: maldonadogarcia@hotmail.com
} 
collected during the period from the first to the 15 of December of 2015 and its analysis was realized by means of the statistical package SPSS version 22. Results: the mean age of the participants was 19.1 years (SD =1.86), the female sex was predominant with $82.0 \%$ (168). A 25.4\% (52) mentioned study and work. $65.3 \%$ of the total sample reported moderate to high levels of stress. The prevalence of alcohol consumption within the last year and within the last month was of $59.0 \%$ and $32.2 \%$ respectively. Some $27.5 \%$ of the total sample reported consumption that indicated dependency, followed by $16.6 \%$ whose level of consumption was harmful. Conclusion: significant levels of stress were found in the university students, as well as an elevated prevalence of alcohol consumption. Moreover, for a significant percentage of those surveyed, their consumption showed signs of dependency, calling for actions directed toward this population to diminish academic stress as well as to give up and/or decrease alcohol consumption.

Descriptors: University Students; Academic Stress; Alcohol Consumption.

\section{RESUMO}

Objetivo: determinar os níveis de estresse escolar e consumo de álcool em calouros universitários. Métodos: estudo descritivo e transversal. Amostragem não probabilística por conveniência, para uma amostra de 205 estudantes de ambos os sexos. Os dados foram coletados durante período de primeiro a 15 de dezembro de 2015 e a análise foi realizada através do SPSS versão 22. Resultados: a idade média dos participantes foi de 19,1 anos (DP $=1,86)$, o sexo feminino predominou com $82,0 \%$. 25,4\% mencionaram estudo e trabalho. $65,3 \%$ do total da amostra relataram níveis de estresse moderado a elevado. A prevalência do consumo de álcool no último ano e no último mês foi de 59,0 e $32,2 \%$, respectivamente. $27,5 \%$ do total da amostra relataram consumo dependente, seguido por $16,6 \%$ do consumo prejudicial. Conclusão: níveis significativos foram identificados na universidade, além de alta prevalência de consumo de álcool. Além disso, uma percentagem significativa dos inquiridos consomem maneira dependente de álcool, o que requer a implementação de intervenções nessa população destinadas a reduzir o stress académico e deixar e/ou reduzir o consumo de álcool.

Descritores: Estudantes Universitários; Estresse Acadêmico; Consumo de Álcool.

\section{INTRODUCCIÓN}

El estrés es uno de los problemas de salud que mayormente afecta a nuestra sociedad actual. El cuál, se haya presente en todos los contextos tanto el laboral, el familiar, como el académico sin importar el género o rango de edad. Se trata de un fenómeno multivariable, en el cual se hace referencia a ciertos acontecimientos o situaciones a los que el individuo se enfrenta, con fuertes demandas que agotan sus recursos de afrontamiento y ponen en riesgo su bienestar ${ }^{1}$.

Lazarus \& Folkman² señalan que el estrés se caracteriza por un conjunto de emociones, sentimientos, pensamientos y dificultades que experimenta el individuo, como resultado de la evaluación que realiza sobre la percepción de las demandas, 
exigencias y de su capacidad para afrontar y atender dichas demandas. Dentro de esta concepción, el estrés se diferencia principalmente de otros estados por su especificidad, está determinado por circunstancias amenazantes particulares del medio ambiente y es una condición con límites temporales que afectan al individuo. Existen factores ambientales generadores de estrés como son aspectos de la organización de la escuela, tareas y actividades académicas, así como el desempeño personal y las relaciones académicas.

Polo et $a^{3}$ definen al estrés académico como aquel que se produce en relación con el ámbito educativo. Orlandilit ${ }^{4}$ señala que cuando una persona está en un proceso de aprendizaje que va desde los grados preescolares hasta la educación universitaria la persona experimenta tensión. En tanto que Barraza $a^{5}$ conceptualiza al estrés académico como un proceso psicológico, sistemático y adaptativo que ocurre en tres momentos; primero, el alumno experimenta en contextos escolares una serie de demandas que el mismo valora como estresantes; segundo, esos estresores provocan un desequilibrio sistémico que se manifiesta en una serie de síntomas y tercero, ese desequilibrio sistémico obliga al alumno a realizar acciones de afrontamiento para restaurar el equilibrio sistémico.

Todo esto como respuesta a las exigencias de adaptación a un mundo en constante evolución y con grandes retos y demandas relacionados con el avance tanto científico como tecnológico. En este sentido, los cambios que viven los jóvenes al enfrentar las nuevas exigencias que les demanda la Universidad, es una situación o experiencia de vida que los pone en riesgo de experimentar estrés.

El estrés se refleja en el grado de reacción o respuesta a los eventos o situaciones académicas que enfrenta el universitario. Por lo tanto, la fuente que origina el estrés académico son todas aquellas nuevas demandas relacionadas con su actividad académica; dichos eventos o problemas se perciben como impredecibles e incontrolables, y por tanto se valoran como estresantes. Diversos autores han identificado que los principales factores generadores de estrés en el medio universitario se relacionaron con el exceso de trabajo académico ${ }^{6}$; falta de tiempo para cumplir con las actividades escolares ${ }^{3}$; presiones políticas y económicas ${ }^{7}$, materias muy demandantes, sobrecarga de actividades escolares, así como el tener que presentar un examen 8 . 
El estrés ocasiona un notable impacto en la salud de la población en general ${ }^{4}$. La literatura identifica múltiples efectos originados por el estrés académico que van desde problemas odontológicos como gingivitis ${ }^{9}$, caries dental ${ }^{10}$, desórdenes mentales ${ }^{11}$, ansiedad, ira y tristez $a^{4}$, depresión ${ }^{12}$ y tendencias suicidas ${ }^{13}$.

De igual forma, diversos autores han vinculado al estrés como factor detonante para el consumo de alcohol, al señalar que la exposición al estrés es una experiencia psicológicamente angustiante y de no contar el sujeto con los recursos o estrategias emocionales para afrontarlo, puede desencadenar el consumo y abuso del alcohol ${ }^{14-17}$. Adicionalmente se puede indicar que el tipo de evento estresante, el tiempo de duración, su frecuencia, durante el curso de la vida en que se presente y la severidad de la tensión experimentada determinará el tipo de consumo o abuso de alcohol.

Según la Organización Mundial de la Salud ${ }^{18}$ el consumo perjudicial de alcohol es especialmente fatal para los grupos más jóvenes, y el alcohol es el principal factor de riesgo a nivel mundial de las muertes de varones entre 15 y 59 años. La ingesta excesiva que a menudo conlleva a comportamientos de riesgo, son ahora prevalentes en diferentes países del mundo como Brasil, Kazajistán, México, Rusia, Sudáfrica y Ucrania entre otros, y desgraciadamente las estadísticas indican que ésta va en aumento.

El alcohol hoy en día es la sustancia psicoactiva de mayor abuso entre los jóvenes a nivel mundial. En el continente europeo más del $90 \%$ de los jóvenes con edades entre 15 y 16 años lo han probado. Un dato importante de señalar es que una de las características del consumo de alcohol entre este colectivo es la tendencia a beber grandes cantidades de una sola vez (5 o más copas por ocasión). Un estudio realizado en el 2003 por la European School Survey Project on Alcohol and Other Drugs reveló que, en 30 de los 35 países europeos analizados, la mayoría de los adolescentes habían realizado consumos masivos hasta alcanzar el estado de embriaguez ${ }^{19}$.

De acuerdo a lo reportado en España por el Plan Nacional sobre Drogas, en 2013 un $76.6 \%$ del total de la población mencionó haber consumido alcohol. La edad de inicio de consumo ocurre entre los 15 y 17 años, mientras que un $62.2 \%$ de los adolescentes españoles declaró haber consumido alcohol alguna vez en su vida ${ }^{20}$. 
El consumo y abuso de alcohol es una práctica muy común en los jóvenes en todo el globo terráqueo y en México no es la excepción, motivo por el cual hoy en día es considerado un problema de salud pública con graves repercusiones para esta población. La Encuesta Nacional de Salud y Nutrición (ENSANUT) ${ }^{21}$, declara que el abuso del alcohol es señalado como la cuarta causa de mortalidad con un $8.4 \%$ del total de las defunciones, puntualizando que sus principales afectaciones son; accidentes vehiculares, patologías derivadas del consumo, lesiones de tipo intencional, no intencional y homicidios.

El Consejo Nacional para la Prevención de Accidentes (CONAPRA) ${ }^{22}$ señala que en México mueren 24 mil personas al año por accidentes vehiculares, la mayoría de ellos atribuibles al consumo abusivo de alcohol; y de los cuales el 28\% son jóvenes con edades de entre los 15 y 29 años. Por lo que los accidentes en vehículo de motor son considerados la primera causa de muerte de las personas en este rango de edad.

En tanto que la Encuesta Nacional de Adicciones ${ }^{23}$ reportó que, en la población adolescente, el consumo de alcohol aumentó significativamente en las tres prevalencias. De tal manera que el consumo alguna vez pasó de $35.6 \%$ a 42.9\%; en el último año de $25.7 \%$ a $30.0 \%$ y en el último mes de $7.1 \%$ a 14.5\%. Esta misma tendencia se observó tanto en los hombres como en las mujeres, especialmente en el consumo del último mes ya que se incrementó en el caso de ellos de $11.5 \%$ a $17.4 \%$ y en ellas de $2.7 \%$ a $11.6 \%$.

Además de que la cerveza sigue siendo la bebida de preferencia entre los jóvenes y de la población total, la consume más de la mitad de la población masculina (53.6\%) y una tercera parte de la población femenina (29.3\%), seguido por los destilados con un porcentaje de consumo de $23.6 \%$ y con un aumento significativo de consumidores adolescentes tanto hombres como mujeres del 2002 a la fecha. De igual modo la ENA (2011) informa que, en cuanto a la edad de inicio de consumo, en el grupo de inicio de los 17 años o menos el porcentaje se incrementó del 2008 de un (49.1\%) al 2011 en un (55.2\%).

En base a lo anteriormente descrito, se identifica la necesidad de explorar sobre la temática del estrés y el consumo de alcohol en universitarios de nuevo ingreso. Por lo que el objetivo del presente estudio fue determinar los niveles de estrés académico y el consumo de alcohol en estudiantes universitarios de nuevo 
ingreso. Los resultados obtenidos, podrán ser tomados en cuenta para estructurar e implementar programas dentro de los campus universitarios encaminados a favorecer la adaptación del universitario de nuevo ingreso, con el fin de evitar o reducir el estrés académico y en consecuencia el consumo de alcohol.

\section{MÉTODOS}

Estudio de tipo descriptivo y transversal. La población estuvo constituida por estudiantes de nuevo ingreso en la licenciatura en Enfermería de la Unidad Académica Multidisciplinaria Matamoros UAT. El muestreo fue no probabilístico por conveniencia, para una muestra total de 205 sujetos de ambos sexos. El criterio de inclusión fue ser alumno de nuevo ingreso (matrícula de agosto de 2015) de la licenciatura en Enfermería de la Unidad Académica Multidisciplinaria Matamoros UAT y estar presente al momento de la aplicación de los instrumentos.

En el presente estudio se utilizó una Cédula de Datos Personales y Prevalencia de Consumo de Alcohol (CDPYPCA), la incluyo cinco reactivos sobre datos biológicos y socioculturales como la edad, género, ocupación, religión y con quien cohabita. En un segundo apartado se indaga la prevalencia de consumo de alcohol alguna vez en la vida, en el último año, en el último mes y en los últimos sietes días.

Para medir el estrés académico se utilizó el Inventario de Estrés Académico (IEA), diseñada por Hernández, Polo y Pozo ${ }^{3}$, el cual mide el grado en que los acontecimientos y circunstancias del estudio son percibidos como estresantes, la escala está conformada por 11 reactivos que incluyen situaciones potencialmente generadoras de estrés dentro del ámbito académico. Para cada una de las situaciones planteadas se presenta una escala con valores de 1 a 5 (donde 1 representa nada de estrés y 5 mucho estrés), que indican el grado de estrés que dicha situación puede generar, un mayor puntaje es indicativo de mayor estrés académico. El nivel de estrés se clasificó de la siguiente forma: bajo nivel de estrés < 25 puntos, moderado nivel de estrés entre 26 y 40 puntos y alto nivel de estrés > 40 puntos.

De igual modo el instrumento recoge información en relación a los tres niveles de respuesta; fisiológico, cognitivo y motor, que el organismo experimenta 
cuando se ve expuesto a situaciones de estrés. La frecuencia de ocurrencia de las respuestas, referidas a los tres niveles de respuesta del organismo, se evalúa en una escala de 1 a 5 , donde 1 significa casi nunca o nunca y 5 significa casi siempre $o$ siempre. La distribución de los elementos quedó conformada de la siguiente forma: nivel fisiológico 4 ítems (2, 5, 8 y 11), nivel cognitivo 5 ítems $(1,4,7,10$ y 12) y nivel motor 3 items (3, 6 y 9). Todo ello permite conocer que situaciones son las que provocan un mayor nivel de estrés, que tipo de respuesta es la que se asocia con tal estrés y cuál es la relación entre cada una de las situaciones y la manifestación conductual. El IEA ha sido utilizado en población española, reportando Alphas de Cronbach que va desde .70 a .80 para cada una de sus dimensiones, además fue validado en población chilena reportando un Alpha de Cronbach de 0.88 para el componente de manifestaciones y un Cronbach de 0.75 para el componente de situaciones académicas estresantes ${ }^{24,25}$.

Para medir el consumo de alcohol se utilizó el Cuestionario de Identificación de Desordenes por Uso de Alcohol (AUDIT) de De la Fuente y Kershenobich ${ }^{26}$, el cuál consta de diez reactivos que examinan el uso de alcohol durante los últimos doce meses y sus consecuencias. Consta de tres dominios, los reactivos del 1 al 3 determinan la cantidad y frecuencia del consumo de alcohol; los reactivos del 4 al 6, exploran la posibilidad de que exista dependencia al alcohol y los reactivos del 7 al 10 el consumo dañino de alcohol. La escala oscila de 0 a 40 puntos, donde a mayor puntaje existe mayor consumo de alcohol. Si se obtiene un valor de 1 a 3 se considera un consumo sin riesgo (consumo sensato), si se obtienen de 4 a 7 puntos se tiene problemas con el consumo (consumo dependiente) y si se registran de 8 a 40 puntos se considera un consumo con riesgo elevado o consumo dañino. Los autores de este instrumento reportaron una sensibilidad de $80 \%$ y una especificidad de $89 \%$. El AUDIT ha sido utilizado en México para medir el consumo de alcohol en adolescentes y en estudiantes universitarios, así como en población chilena. En estos estudios se ha reportado un Alpha de Cronbach de .77, .84 y .84 respectivamente ${ }^{1427,28}$.

Primeramente, se solicitó la autorización de la dirección de la Unidad Académica Multidisciplinaria Matamoros UAT, así como la autorización del Comité de ética e Investigación de la unidad. Para la selección de los participantes se contó 
con las listas de asistencia de los alumnos de nuevo ingreso de la licenciatura en enfermería y fueron tomados en cuenta el total de los alumnos registrados.

La recolección de los datos se llevó a cabo en una de las aulas. Se buscó un espacio de tiempo en el cual no interfiriera con sus actividades escolares, el tiempo aproximado para el llenado de los instrumentos fue de 30 minutos. Antes de iniciar la recolección de datos se explicó el propósito de la investigación, se solicitó su participación voluntaria mediante el consentimiento informado por escrito, y se hizo de su conocimiento que la información sería confidencial, respetándose el anonimato. Se le indicó que, si no deseaba participar, se respetaría su decisión y no habría ninguna repercusión en su situación escolar. Posterior a la firma del consentimiento se le entregó a cada estudiante un sobre cerrado conteniendo la cédula de datos personales y los dos instrumentos para que procediera con el llenado, se indicó que, al término del llenado de los instrumentos, los volvieran a depositar en el sobre, lo cerraran y lo depositaran en una caja forrada que se ubicó al frente del aula. Por último, se les agradeció su participación en el estudio.

El estudio fue aprobado por el Comité de Ética e Investigación de la Unidad Académica Multidisciplinaria Matamoros UAT y se apegó a lo dispuesto por la Ley General de Salud en Materia de Investigación en el área de la salud ${ }^{29}$. Se respetó la dignidad de los sujetos mediante un trato respetuoso y profesional, así como la protección de sus derechos humanos. Además, se contó con el consentimiento informado por escrito del participante y se garantizó el anonimato y la confidencialidad de la información relacionada con su privacidad, de igual forma se les informó que tendrían la oportunidad de retirar su consentimiento informado cuando así lo desearan.

Los datos obtenidos de la recolección fueron procesados a través del programa estadístico SPSS versión 22.0, para el análisis de los datos se utilizó estadística descriptiva para frecuencias y porcentajes.

\section{RESULTADOS}

La población del estudio estuvo conformada por 205 estudiantes de ambos sexos, la media de edad de los participantes fue de 19.1 años ( $D E=1.86)$, con una mínima de 17 y una máxima de 28 años respectivamente. Predominó el sexo 
femenino con un $82.0 \%$ (168). Un $25.4 \%$ (52) mencionó estudiar y trabajar; la religión que mas profesan es la católica 70.7\% (154), seguida de la cristiana 17. 1\% (35). El $80 \%$ (164) vive con sus padres, mientras que solo un $4.9 \%$ (10) de los encuestados refirió vivir actualmente con su pareja.

En relación a los niveles de estrés, predominó el nivel de estrés moderado $58.5 \%$ (120), seguido por el nivel bajo 34.6\% (71); mientras que solo un 6.9\% (14) de la población reportó alto nivel de estrés (Tabla 1). Las principales situaciones generadoras del estrés académico fueron la sobrecarga académica 48.3\%, seguida de falta de tiempo para cumplir con las actividades escolares, y un tercer detonante fue la realización de trabajos obligatorios para aprobar la asignatura 38.6\%.

En lo que respecta a la prevalencia de consumo, se encontró que el $78.0 \%$ (160) de los universitarios han consumido alcohol alguna vez en la vida, un $59.0 \%$ (121) continuó con el consumo en el último año, el 32.2\% (66) lo hizo en el último mes; mientras que solo el $11.2 \%$ (23) refirió haber consumido alcohol en la última semana (Tabla 2).

Adicionalmente se encontró que el $26.3 \%$ (54) de los universitarios consumidores indicaron que en un día típico consumen de 1 a 3 bebidas alcohólicas, mientras que el $2.9 \%$ consume 10 o más bebidas por ocasión. En lo referente al tipo de bebidas que más consumen predominó la opción cerveza 56 (27.3\%), seguido por las bebidas preparadas 71 (34.6\%), en tanto que el whisky representó un $4.9 \%$ con un total de 10 menciones (Tabla 3).

En cuanto a los tipos de consumo, el mayor porcentaje de los universitarios mencionó consumir de forma sensata $56.2 \%$ (88), seguido del consumo dependiente $27.3 \%$ (43) y un importante porcentaje de los encuestados señalo hacerlo de forma dañina 16.5\% (26) (Tabla 4).

Tabla 1. Nivel de estrés académico.

\begin{tabular}{lrc}
\hline Nivel de estrés & $f$ & $\%$ \\
\hline Bajo nivel de estrés & 71 & 34.6 \\
Moderado nivel de estrés & 120 & 58.5 \\
Alto nivel de estrés & 14 & 6.9 \\
Total & 205 & 100.0 \\
\hline
\end{tabular}

Nota: $f=$ frecuencia, $\%=$ porcentaje $n=205$ 
Tabla 2. Prevalencia de consumo de alcohol

\begin{tabular}{lcccc}
\hline \multirow{2}{*}{ Prevalencia } & $f$ & $\%$ & LI & IC 95\% \\
\cline { 4 - 5 } & & & 1.18 & 1.29 \\
Últguna vez & 160 & 78.0 & 1.34 & 1.48 \\
Último año & 121 & 59.0 & 1.61 & 1.74 \\
Último semana & 66 & 32.2 & 1.84 & 1.93 \\
\hline
\end{tabular}

Nota: $f=$ frecuencia, $\%$ = porcentaje, IC = Intervalo de Confianza, LI = Límite Inferior, LS = Límite Superior

Tabla 3. Tipos de bebidas preferidas de los participantes.

\begin{tabular}{lrc} 
& $f$ & $\%$ \\
\hline Cerveza & 56 & 27.3 \\
Bebidas preparadas & 71 & 34.6 \\
Whisky & 10 & 4.9 \\
Vino & 2 & 1.2 \\
Otros & 18 & 8.8 \\
\hline Nota: $f=$ frecuencia, \% = porcentaje, $n=157$ & &
\end{tabular}

Tabla 4. Tipos de consumo.

\begin{tabular}{lcc}
\hline & $f$ & $\%$ \\
\hline Consumo sensato & 88 & 56.2 \\
Consumo dependiente & 43 & 27.3 \\
Consumo dañino & 26 & 16.5 \\
Total & 157 & 100.0 \\
\hline Nota: $f=$ frecuencia, \% porcentaje, $n=157$ & &
\end{tabular}

\section{DISCUSIÓN}

El presente estudio permitió determinar los niveles de estrés académico, la prevalencia y los tipos de consumo de alcohol en estudiantes de nuevo ingreso al ámbito universitario, la muestra estuvo constituida por 205 participantes de una universidad pública de la ciudad de H. Matamoros Tamaulipas, México.

El ingreso a la universidad es un proceso que para muchos resulta ser muy demandante, ya que implica llegar a un nuevo entorno, además de cumplir con todas las exigencias que implica el mantenerse como alumno regular y aprobar el total de los créditos. En el presente estudio, se evidenció que más de la mitad de los universitarios encuestados presentan niveles de estrés de moderado a alto, Journal Health NPEPS. 2017; 2(1):133-147. 
datos similares a lo reportado en un estudio realizado en estudiantes de nueva incorporación a la universidad en Valencia España, donde encontraron que un $82 \%$ de los participantes manifestaron haber experimentado de mucho a bastante estrés ${ }^{24}$.

En cuanto a las fuentes generadoras de estrés, la causa principal resultó ser la sobrecarga académica $48.3 \%$, seguida de falta de tiempo para cumplir con las actividades escolares, y un tercer detonante fue la realización de trabajos obligatorios para aprobar la asignatura 38.6\%; dichos resultados coinciden con lo reportado por Tam y Dos Santos ${ }^{8}$ y Díaz .

En lo referente al consumo de alcohol, los resultados obtenidos en el presente estudio arrojaron que el $78 \%$ de los encuestados ha consumido alcohol alguna vez en la vida; dicho porcentaje supera las prevalencias de consumo de alcohol para esta población reportado por la ENA, 201123. Sin embargo, dichos hallazgos son similares a lo reportado por Palacios ${ }^{30}$; Castaño, García y Marzo ${ }^{31}$; Zurita y Álvaro ${ }^{20}$. En tanto que el $59.0 \%$ (121) de la muestra reporto haber consumido alcohol en el último año, el 32.2\% (66) lo hizo en el último mes; mientras que solo el $11.2 \%$ (23) refirió haber consumido alcohol en la última semana; la disminución de la frecuencia de consumo puede deberse a una mayor adaptabilidad al estrés que genera el cursar una carrera universitaria, así como a un mayor conocimiento y concientización sobre las consecuencias o complicaciones derivadas del consumo de alcohol ${ }^{32}$.

Un dato importante de resaltar es que un $2.9 \%$ de los jóvenes reportaron consumir de tipo "binge drinking", es decir consumir 10 o más bebidas por ocasión y llegar a un estado de embriaguez. Mientras que un $27.3 \%$ de los universitarios identifican a la cerveza como su bebida de preferencia, dato que es coherente con lo reportado en la ENA, 2011 y en el estudio de Arévalo et al ${ }^{33}$.

En relación a los tipos de consumo, la mayor proporción de los participantes en el estudio (55.7\%), se ubican en el tipo de consumo sensato; cifra que coincide con lo señalado por Puig et al ${ }^{34}$, quienes en una muestra de estudiantes mexicanos encontraron que un $54.0 \%$ menciono consumir con un patrón de bajo riesgo. Sin embargo, en el presente estudio un alarmante porcentaje (16.6\%) señaló tener un tipo de consumo dañino.

\section{CONCLUSIÓN}


En base a lo descrito, se concluye que el ingreso a la universidad es una etapa de transición de la vida que predispone un estado de vulnerabilidad en los jóvenes, por lo cual se considera importante implementar en el ámbito universitario programas dirigidos a la prevención y disminución del estrés académico, que eviten que el joven afronte el estrés de manera inadecuada desencadenando el uso y abuso del alcohol, afectando su salud y el rendimiento académico. Así como estructurar e implementar intervenciones breves (asesoría, talleres, foros) dirigidas a la prevención y/o disminución del consumo de alcohol en jóvenes universitarios, con el objetivo de que los universitarios lleven un estilo de vida saludable que se refleje en su bienestar.

\section{REFERENCIAS}

1. Pulido RMA, Serrano SML, Valdés CE, Chávez MMT, Hidalgo MP, Vera GF. Estrés académico en estudiantes universitarios. Psicol salud. 2011; 21(1):31-37.

2. Lazarus RS, Folkman S. Estrés y procesos cognitivos. Barcelona: Martínez Roca; 1986.

3. Polo A, Hernández JM, Pozo C. Evaluación del Estrés Académico en Estudiantes Universitarios. Ansiedad estrés. 1996; 2(2/3):159-72.

4. Orlandini A. El estrés, qué es y cómo evitarlo. $5^{\mathrm{a}}$ ed. México: Fondo de Cultura Económica; 2012.

5. Barraza MA. Características del estrés académico en los alumnos de educación media superior. Rev electrónica psicol cient. 2005. Disponible en: http://www.psicologiacientifica.com/bv/psicologia-167-1-cientifica.html

6. Díaz MY. Estrés académico y afrontamiento en estudiantes de medicina. Rev Hum Méd. 2010; 10(1):85-93.

7. Maruris RM, Cortéz GP, Cabañas GMA, Godínez JF, González RM, Landero HR. Factores estresantes asociados al hiperestrés en estudiantes universitarios de la ciudad de Chilpancingo, Guerrero, México. Summa Psicol UST. 2012; 9(1):15-21.

8. Tam PE, Santos CB. El consumo de alcohol y el estrés entre estudiantes del segundo año de enfermería. Rev Latinoam Enferm. 2010;18:493-503. 
9. Johannsen A, Bjurshammar N, Gustafsson A. The influence of academic stress on gingival inflammation. Int J Dent Hygiene. 2010; 8:22-27.

10. Mejía RC, Alanís TJ, Argueta FL, Legorreta RA. Academic stress as a risk factor for dental caries. Inter dent j. 2012; 62:127-131.

11. Ekpenyong CE, Davis KJ, Akpan UP, Daniel NE. Academic stress and menstrual disorders among female undergraduates in Uyo, South Eastern Nigeria - the need for health education, Niger. J. Physiol. Sci.201; 26:193-198.

12. Jayanthi P, Thirunavukarasu M, Rajamanickam R. Academic stress and depression among Adolescents: A Cross-sectional Study. Indian pediatr. 2014; 52:217-219.

13. Venumadhava GS, Mayuri S. Academic stress and suicidal tendency. Golden Research Toughts. 2014; 3(11):1-10.

14. Armendáriz GNA, Villar LMA, Alonso CMM, Alonso CBA, Oliva RNN. Eventos estresantes y su relación con el consumo de alcohol en estudiantes universitarios. Investig. Enferm. Imagen desarr. 2012; 14(2):97-112.

15. Barnes GE. Personality, life stress, alcohol consumption and alcohol problems in Vancouver couples. Procedia Social and Behavioral Sciences. 2013; 84:1464-1468.

16. Keyes KM, Hatzenbuehler ML, Hasin DS. Stressful life experiences, alcohol consumption, and alcohol use disorders: the epidemiologic evidence for four main types of stressors. Psychopharmacol. 2011; 218:1-17.

17. Kim HJ, Martins SS, Shmulewitz D, Santaella J, Wall MM, Keyes MK, Eaton RN, Krueger R, Grant FB, Hasin SD. Childhood maltreatment, stressful life events, and alcohol craving in adult drinkers. Alcohol Clin Exp Res. 2014;38(7):2048-2055.

18. Organización Mundial de la Salud. (2014). Recuperado de http: //www.docsalud.com

19. Salamó AA, Gras PME, Font-Mayolas S. Patrones de consumo de alcohol en la adolescencia. Psicothema. 2010; 22(2):189-195

20. Zurita F, Álvaro Jl. Repercusión del tabaco y alcohol sobre factores académicos y familiares en adolescentes. Health and Addictions. 2014; 14(1):59-70.

21. Encuesta Nacional de Salud y Nutrición 2012. Disponible en: http://ensanut.insp.mx/

22. Consejo Nacional para la Prevención de Accidentes 2015. Disponible en: http: //conapra.salud.gob.mx/ 
23. Encuesta Nacional de Adicciones (ENA, 2011). Disponible en: http: //www.conadic.salud.gob.mx/interior/ena_.html

24. García RR, Pérez GF, Pérez BJ, Natividad LA. Evaluación del estrés académico en estudiantes de nueva incorporación a la universidad. Rev latinoam psicol. 2012; 44(2):143-154.

25. Herrera AD, Rodríguez RM, Valverde TM. Validación del Inventario de Estrés Académico de Polo, Hernández y Pozo en Estudiantes Universitarios de la Comuna de Concepción. Disponible

en: https: //spamelarf.files.wordpress.com/2010/09/cuanti2.pdf

26. De la Fuente JR, Kershnobich D. El alcoholismo como problema médico. Rev fac med UNAM. 1992; 35(2):47-51.

27. Castillo CA, Pizarro E, Luengo D, Soto BG. Consumo de alcohol y autoinforme de eventos violentos en Chile. Adicciones. 2014; 26(1):46-53.

28. Villegas PMA, Alonso CMM, Alonso CBA, Guzmán FFR. Eventos estresantes y la relación con el consumo de tabaco y alcohol. Cienc enferm. 2014; 20(1):35-46.

29. Secretaría de Salud. Reglamento de la Ley General de Salud en Materia de Investigación para la Salud. Cd de México: Editorial Porrúa; 1987.

30. Palacios DJR. Exploración de los motivos para consumir alcohol en adolescentes. Psicol iberoam. 2012; 20(1):29-39.

31. Castaño PGA, García CJA, Marzo CDC. Consumo de alcohol y factores intervinientes en estudiantes universitarios. Rev Cuba Salud Pública. 2014; 40(1):4754.

32. Valle R, Sánchez E, Perales A. Sintomatología depresiva y problemas relacionados al consumo de alcohol durante la formación académica de estudiantes de medicina. Rev Perú Med Exp Salud Publica. 2013; 30(1):54-57.

33. Arévalo TLL, Díaz CAJ, González MFD, Simancas PMA. Consumo de bebidas alcohólicas y factores relacionados en estudiantes de odontología. Rev clín med fam. 2010; 3(2):93-98.

34. Puig NA, Cortaza RL, Pillon SC. Consumo de alcohol entre estudiantes mexicanos de medicina. Rev Latinoam Enferm. 2011; 19:714-721. 


\section{Agradecimientos}

Agradecimiento a la Comisión Interamericana para el Control del Abuso de Drogas/CICAD de la Secretaria de Seguridad Multidimensional/SSM de la Organización de los Estados Americanos/OEA, la Secretaria Nacional de Políticas sobre Drogas/SENAD do Gabinete de Seguridad Institucional/Brasil, la Escuela de Enfermería de Ribeirao Preto de la Universidad de Sao Paulo y Centro Colaborador de la Organización Mundial de la Salud para el Desarrollo de la Investigación en Enfermería, la población representada en los estudios de investigación, bien como a las autoridades de las Universidades representadas por los participantes del Curso de Formación a Distancia de Investigadores sobre Alcohol y Otras Drogas Psicoactivas.

Conflito de interesses: Os autores declaram não haver conflito de interesses.

Como citar este artigo: García LH, Gherardi-Donato ECS, Castillo MMA, Silva RC, García PG, García VM. Estrés académico y consumo de alcohol en universitarios de nuevo ingreso. Journal Health NPEPS. 2017; 2(1):133-147. 